ESJ Natural/Life/Medical Sciences

\title{
Modélisation Des Niveaux D’eau Extrêmes Sur Les Plages Sableuses De Malibu, De Gadaye Et De Malika (Dakar, Sénégal) Consécutive À La Tempête Du 19 Novembre 2018
}

\section{Cheikh Omar Tidjani Cisse,}

Université Gaston Berger, Laboratoire Leïdi "Dynamique des Territoires et Développement”, Saint-Louis, Sénégal

\section{Papa Sagne,}

Kader BA,

Laboratoire de Biostratigraphie-Sédimentologie, Département de Géologie, Université Cheikh Anta Diop de Dakar (Sénégal).

Laboratoire de Télédétection Appliquée, Institut des Sciences de la Terre, Université Cheikh Anta Diop de Dakar (Sénégal).

\section{Boubacar Fall,}

Laboratoire de Biostratigraphie-Sédimentologie, Département de Géologie, Université Cheikh Anta Diop de Dakar (Sénégal).

\section{Boubou Aldiouma SY,}

Université Gaston Berger, Laboratoire Leïdi "Dynamique des Territoires et Développement”, BP 234 Saint-Louis, Sénégal

\section{Augustin Marone}

Laboratoire LETG-Géophen "Littoral-Environnement-Télédétection-

Géomatique-Géographie physique et environnement”, Caen

Doi:10.19044/esj.2022.v18n3p79

Submitted: 06 December 2021

Accepted: 24 January 2022

Published: 31 January 2022
Copyright 2022 Author(s)

Under Creative Commons BY-NC-ND 4.0 OPEN ACCESS

Cite As:

Cisse T.O.C., Sagne P., Kader B.A.,Fall B., Aldiouma B., \& Marone A., (2022). Modélisation des niveaux d'eau extrêmes sur les plages sableuses de Malibu, de Gadaye et de Malika (Dakar, Sénégal) consécutive à la tempête du 19 novembre 2018 European Scientific Journal, ESJ, 18 (3), 1.

https://doi.org/10.19044/esj.2022.v18n3p79

\section{Abstract}

Dans un contexte mondial de réchauffement climatique, les espaces côtiers sont de plus en plus menacés par les risques de submersion. La modélisation de ces submersions marines, compte tenu du danger qu'elles 
représentent, constitue une étape importante avant toute initiative de protection côtière des zones à fort enjeux socio-économiques.

L'objectif de cette contribution est de faire une modélisation des niveaux d'eau extrêmes sur les plages sableuses de Malibu, de Gadaye et de Malika à partir de la tempête du 19 novembre 2018 à Dakar. Pour ce faire, un modèle empirique a été utilisé pour reconstituer l'extension spatiale de l'onde de tempête sur les plages. Les résultats révèlent d'une part, que les niveaux d'eau extrêmes sont sensiblement les mêmes sur les trois plages, et d'autre part, que ces niveaux extrêmes n'ont pas excédé la crête maximale de trait de côte des trois plages. Quand bien même l'extension spatiale du jet de rive (runup et setup) sur les plages est sensiblement la même, la sensibilité des plages à cet évènement morphogène est différente où des formes d'ablation (microfalaise) ont été observées sur la partie haute de Gadaye et Malika. Cela peut être expliqué par les différentes caractéristiques morphologiques des plages un mois avant la tempête, en octobre 2018. En définitive, les résultats laissent apparaître clairement une hétérogénéité dans les ajustements morphologiques et sédimentaires trois mois après le passage de la tempête de février 2019. Toutefois, une certaine résilience de la zone est notée en raison de la présence de dunes qu'il faut impérativement éviter leur dénaturalisation pour une protection naturelle et durable de la zone.

Keywords: Modélisation, Onde De Tempête, Niveaux D’eau Extrêmes, Crête Maximale, Jet De Rive, Morphogène, Ablation 


\title{
Modelling Of Extreme Water Levels On Malibu, Gadaye And Malika Sandy Beaches (Dakar, Senegal) Following The 19 November 2018
}

\author{
Cheikh Omar Tidjani Cisse, \\ Université Gaston Berger, Laboratoire Leïdi "Dynamique des Territoires et \\ Développement”, Saint-Louis, Sénégal
}

\section{Papa Sagne,} Kader BA,

Laboratoire de Biostratigraphie-Sédimentologie, Département de Géologie, Université Cheikh Anta Diop de Dakar (Sénégal).

Laboratoire de Télédétection Appliquée, Institut des Sciences de la Terre,

Université Cheikh Anta Diop de Dakar (Sénégal).

\section{Boubacar Fall,}

Laboratoire de Biostratigraphie-Sédimentologie, Département de Géologie,

Université Cheikh Anta Diop de Dakar (Sénégal).

\section{Boubou Aldiouma SY,}

Université Gaston Berger, Laboratoire Leïdi “Dynamique des Territoires et Développement”, BP 234 Saint-Louis, Sénégal

\section{Augustin Marone}

Laboratoire LETG-Géophen “Littoral-Environnement-Télédétection-

Géomatique-Géographie physique et environnement”, Caen

\section{Abstract:}

In a global context of global warming, coastal areas are increasingly threatened by the risk of flooding. The modeling of these marine submersions, because of the danger they represent, is an important step before any coastal protection initiative for areas with high socio-economic stakes.

The objective of this scientific contribution is to model the extreme water levels on the sandy beaches of Malibu, Gadaye and Malika from the storm of 19 November 2018 in Dakar. To do so, an empirical model was used to reconstruct the spatial extension of the storm surge on the beaches. The results show that the modeled extreme water levels are approximately the same on the three beaches, and that their extreme levels did not exceed the maximum coastline crest of the three targeted beaches. Even though the spatial extension of the run-up on the beaches is almost homogeneous, the sensitivity of the beaches to this morphogenic event is different or forms of ablation (micro-cliffs) have been observed on the upper part of Gadaye and Malika beaches. This can be explained by the different morphological characteristics of the beaches one month before the storm, in October 2018. Finally, the results clearly show heterogeneity in the morphological and sedimentary 
adjustments three months after the storm of February 2019. However, a certain resilience of the area is noted due to the presence of dunes that must be avoided for a natural and sustainable protection of the area.

Keywords: Modeling, Storm Surge, Extreme Water Levels, Maximum Coastline Crest, Run Up, Morphogenic, Ablation

\section{Introduction}

Dans un contexte mondial marqué par le changement climatique conduisant à l'élévation du niveau marin de l'ordre de $30 \mathrm{~cm}$ tous les 100 ans (GIEC, 2007), les espaces côtiers sont de plus en plus menacés par les risques d'érosion et de submersion marine qui sont parmi les conséquences prévisibles de ce phénomène (Gozannet et al. 2015). Selon le GIEC (2013), une élévation du niveau de la mer de $0,5 \mathrm{~m}$ engendrerait une augmentation de 10 à 100 fois de la fréquence des submersions marines dans le monde. Ces dernières, résultant de la conjonction d'une faible pression atmosphérique et d'un « fort vent d'afflux » (Letortu et al., 2012), deviennent une véritable problématique environnementale pour les littoraux soumis à de fortes pressions humaines. Ces submersions sont parmi les aléas météorologiques les plus dévastateurs le long des traits de côte (Balouin et al., 2011).

En Afrique de l'Ouest, elles constituent une préoccupation fondamentale. Vu leur ouverture sur la façade Atlantique, la plupart des côtes ouest-africaines sont exposées à la submersion (Tchindjang et al., 2019).

Le littoral sénégalais qui s'étend sur environ $718 \mathrm{~km}$ de côte est souvent soumis à des épisodes météo-marins extrêmes. Il est très sensible à l'érosion et à la submersion marine du fait de son faible dénivelé et de la nature sableuse d'une partie de ces côtes (Weissenberger et al., 2016). La fréquence de ces épisodes extrêmes en sus de l'érosion consécutive aux Hautes et Basses marées, a des effets dévastateurs sur les littoraux sableux. En effet, les submersions marines enregistrées sur ce littoral en juillet 1993, août 2004, juillet 2005, juillet 2007 et mai 2014 (Ndour, 2015) confirment sa vulnérabilité face à ce risque côtier. Ces submersions, au-delà de leurs conséquences socioéconomiques, ont des impacts non négligeables sur la dynamique des plages sableuses. Selon Gervais et al. (2010), les tempêtes sont des évènements brutaux entrainant souvent des impacts morphologiques rapides et importants parfois même irréversibles sur les plages sableuses. Ces submersions marines surviennent lors de conditions météorologiques défavorables provoquant une élévation anormale du niveau marin. Selon Anselme et al. (2008), cette élévation du niveau marin se traduit sur les littoraux à topographie basse par la conjonction de deux phénomènes : le setup et le runup. D’après Garcin et al. (2009), le setup et le runup sont responsables de la submersion marine lors d'une tempête. Lorsque les conditions météorologiques sont défavorables 
(baisse de la pression atmosphérique), les vents d'afflux poussent les masses d'eau à la côte faisant que le niveau marin devient plus important au niveau de la marée astronomique (Anselme et al., 2008). Selon Bulteau et al. (2012), la caractéristique principale d'une submersion marine est le niveau maximal atteint à la côte pendant l'épisode de tempête. Cette surélévation du plan d'eau à la côte provoque une extension de la limite spatiale du jet de rive (runup et setup) en condition normale. Durant les évènements tempétueux, la surcote provoque sur les côtes sableuses une amplification de l'impact des vagues abordant la côte (Balouin et al., 2011). En effet, l'action des vagues déferlantes se traduit par le run up qui correspond à la hauteur des vagues lorsqu'elles abordent la plage (Jeuck, 2018). Il constitue un élément important dans l'évaluation des impacts observés sur les plages après une submersion ou tempête marine (Robin, 2013). Selon Enjalbert et al. (2012), le run un est le principal processus impliqué dans l'érosion du haut de plage ainsi que des problèmes liés à la submersion marine.

Actuellement, l'essentiel des productions scientifiques sur le littoral sénégalais s'intéresse généralement aux aspects géomorphologiques, leur évolution spatio-temporelle, les impacts des aménagements côtiers sur l'évolution morphosédimentaire (Faye, 2010 ; Thior et al., 2019) Cependant, les études abordant la submersion marine ainsi que leurs modélisations ne sont pas encore véritablement initiées. Or, cet aléa joue un rôle important dans l'évolution morphologique des plages.

La modélisation des submersions marines des plages, compte tenu du danger qu'elles représentent, constitue une étape importante avant toute initiative de protection côtière des zones à fort enjeux socio-économique (Arnaud et Bertin, 2014). Ainsi, le présent article vise à modéliser les niveaux d'eau extrêmes enregistrés sur les plages sableuses de Malibu, Gadaye et Malika de la région de Dakar lors de la tempête du 19 novembre 2018.

Plus précisément, cette quantification des niveaux d'eau extrêmes permet de bien poser la problématique de la vulnérabilité des plages sableuses de Dakar à la submersion marine et leur résilience face à l'action des vagues de tempête.

\section{Présentation de la zone d'étude}

Cette étude concerne le littoral Nord de Dakar plus particulièrement les plages de Malibu (Golf Sud), Gadaye (Guédiawaye) et Malika (figure 1) situées sur un même linéaire côtier. Ces plages, de nature sableuse, constituent des milieux fragiles et très sensibles à l'action des agents hydrodynamiques (Paskoff, 1993, 1998) qui entraine des variations morphologiques à différentes échelles temporelles (journalières, saisonnières, annuelles, pluriannuelles...) selon les conditions du milieu (Sagne, 2021). La quantité de sédiments ainsi perdue lorsqu'une houle érosive se produit ne peut être compensée que si elles 
disposent d'importants stocks de sédiments, en général, constitués par les dunes (Paskoff, 1993 ; Pinot, 1998). Cette disposition, d'ailleurs notée dans la zone, pourrait constituer un élément de résilience géologique à ce niveau du littoral Dakar.

Du point de vue topographique, les altitudes les plus élevées sur la côte Nord de Dakar sont notées à Yoff. Elles varient dans cette partie entre 20 et 40 m (Sagne, 2021). Cependant, elles diminuent en allant vers le Lac Rose située à l'est. Dans la zone de Malibu à Malika, elles dépassent rarement $20 \mathrm{~m}$ (Sagne, 2021). Elles sont plus élevées au niveau de la plage de Malibu et devient plus basses en allant vers Malika où les valeurs maximales tournent autour de $15 \mathrm{~m}$ (Sagne, 2021). Cette situation serait un élément d'exposition majeur de cette partie aux submersions marines liées aux fortes houles et à l'élévation du niveau de la mer induite par le changement climatique.

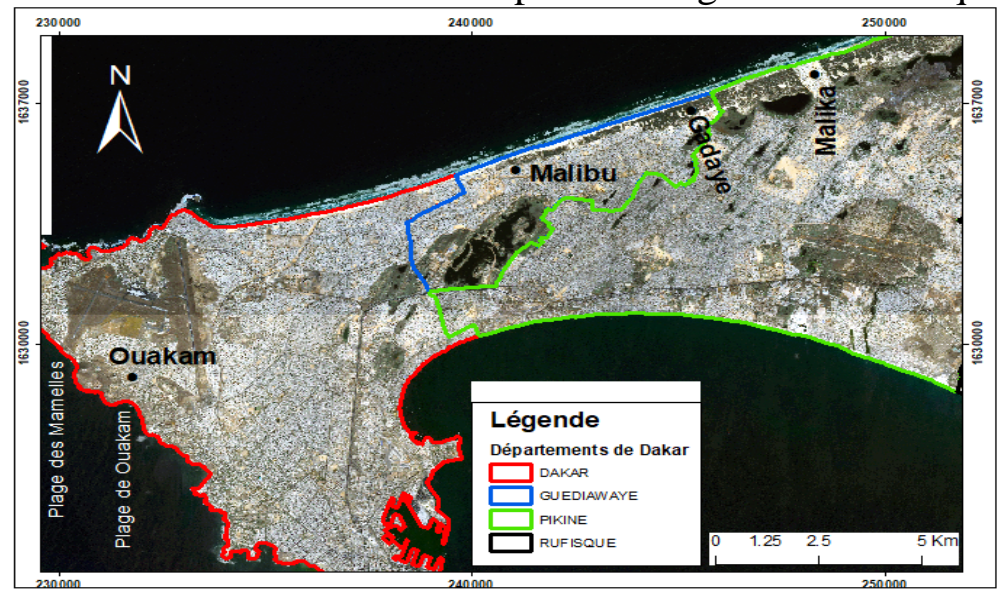

Figure 1 : Situation géographique de la zone d'étude

Par ailleurs, la zone d'étude est principalement sous l'influence des houles du nord-ouest qui constituent les principaux agents responsables de sa dynamique morpholo-sédimentaire puisqu'elle est protégée des houles du sud-ouest par la pointe des Almadies (Niang-Diop, 1995 ; Ndour, 2015). A cela s'ajoutent le faible marnage (inférieur à $2 \mathrm{~m}$ ) lui offrant le caractère de côte micro-tidale, et les vitesses des courants de marées qui sont inférieures à $0,15 \mathrm{~m} / \mathrm{s}$ indiquant une faible influence de la marée sur les mouvements de cette plage (Fall, 2004 ; Ndour, 2015 ; Stéphanain et al., 2011). L'influence des houles exceptionnelles sur la dynamique de cette partie n'est pas à négliger puisqu'elles occasionnent des phénomènes d'érosion rapide susceptible d'atteindre le pied des dunes où des quantités importantes de sédiments sont érodées (Sagne et al., 2020). 


\section{Matériels et méthodes}

\subsection{Données météorologiques et océanographiques}

Les données de réanalyse de la houle ont été obtenues de l'Agence National de l'Aviation Civile et de la Météorologie (ANACIM) pour les caractéristiques hydrodynamiques de la tempête. Cependant, celles du marégraphe de Dakar (capteur Ra2 de l’Université de Hawaï) ont été obtenues du Service Hydrographique des Phares et Balises. Ces données ont par la suite été corrigées avec la constante de 0,06 m. Les données de marée prédite utilisées sont celles de SHOM (Service Hydrographique de la Marine). Ces dernières sont comparables aux données du marégraphe de Dakar puisqu'elles ont le même référentiel, qui est le zéro hydrographique. Le niveau marin observé au marégraphe de Dakar est converti en données géodésiques pour une superposition rigoureuse avec les profils de plage. A cet effet, le modèle de Wöppelmann et al. (2008), figure (3) est utilisé pour convertir les niveaux de marée en données géodésiques. Selon Wöppelmann et al. (2008), le zéro hydrographique et le datum vertical sont séparés par une distance de 0,981 m (2,301 m-1,320 m), (figure 2), qui correspond à la valeur géodésique des niveaux d'eau de référence, ce qu'illustre le diagramme des repères et des points de référence pertinents de Wöppelmann et al. (2008)

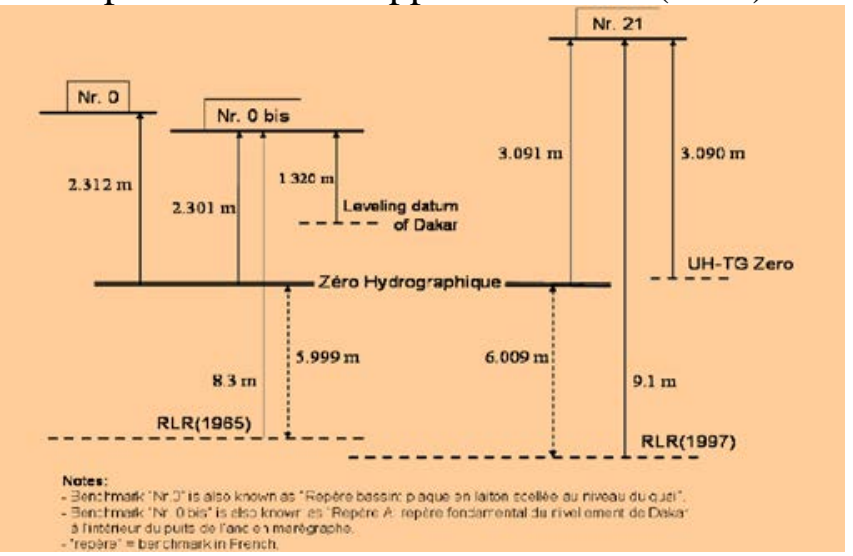

Figure 2: Situation des repères de marée et de points de référence pertinents Wöppelmann et al. (2008).

\subsection{Données topographiques}

Cette recherche est également basée sur des levés topographiques pour mesurer la morphologie du segment littoral faisant l'objet de cette contribution par une série de profils perpendiculaires à la ligne de rivage, depuis le pied des dunes jusqu'à la zone de déferlement. Ainsi, trois profils de plage ont été levés respectivement au niveau des plages de Malibu, Gadaye et Malika (figure 3) aux mois d'octobre 2018 (avant le passage des houles) et de novembre 2018 (juste après leur passage). Après ces deux missions, une autre campagne de 
collecte a été réalisée en février 2019 pour évaluer le temps de retour des sédiments mobilisés par cet évènement.

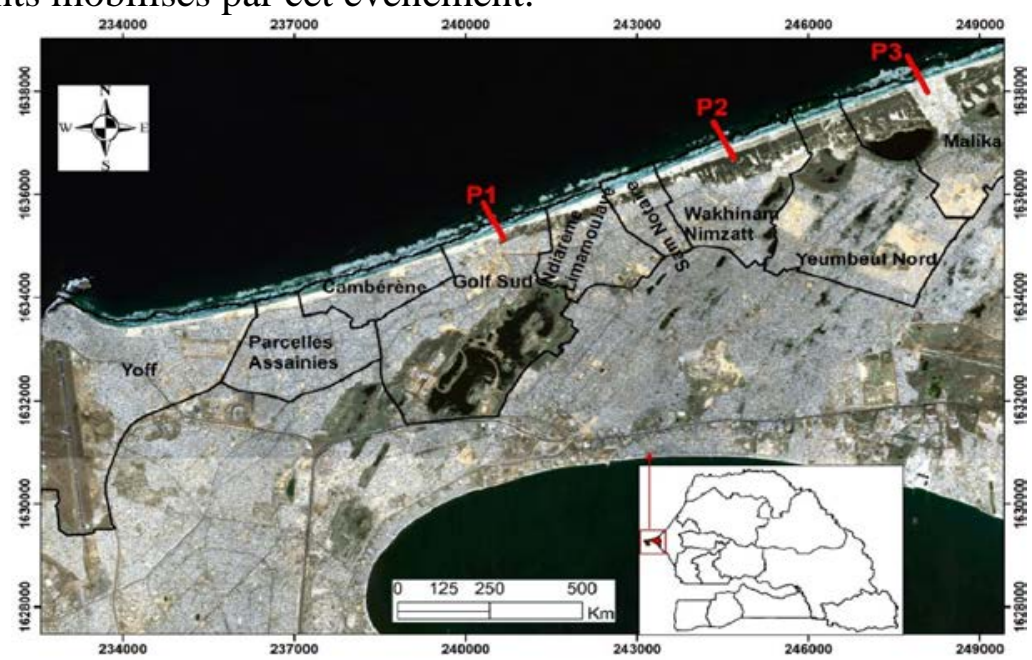

Figure 3: Position des sites échantillons dans le secteur d'étude

Les profils ont été levés avec un matériel constitué par un niveau de chantier, un trépied, une mire, un ruban, une boussole et un GPS. Les coordonnées des repères arrière fixes à partir desquels les profils ont été calés sont déterminées par le GPS. La boussole permet de contrôler la direction des profils pour permettre leur comparaison rigoureuse. Pour mieux respecter la morphologie de chaque profil durant les levés topographiques, les points de rupture de pente minutieusement mesurés.

\subsection{Analyse des niveaux d'eau extrêmes de la tempête du}

\section{8 et 19 novembre 2018}

Dans le cadre de ce travail, l'estimation des niveaux d'eau extrême repose sur l'utilisation des modèles empiriques pour quantifier le run up ou la limite maximale atteint par les vagues lors de la tempête. Ce run up revêt un caractère important dans l'étude des niveaux d'eau extrêmes. Selon Enjalbert et al. (2012), le run up est le principal processus impliqué dans l'érosion du haut de plage ainsi que des problèmes liés à la submersion marine. Ce travail repose sur la combinaison de deux paramètres : l'action de la houle sur la côte traduit par le runup et le niveau marin maximal observé au marégraphe de Dakar. Selon Caspar et al. (2007), les submersions induites par les tempêtes résultent la plupart du temps de la conjonction d'une surcote à la marée et une forte agitation marine. D'après Arnoux et al. (2018), le runup peut facilement s'additionner au niveau marin de manière à représenter physiquement les hauteurs atteintes par un état de mer donné. 
Les niveaux d'eau extrême lors de la tempête du 19 novembre 2018 ont été estimés en additionnant la valeur du runup obtenue à travers le modèle empirique et le niveau marin maximal observé au marégraphe de Dakar.

L'utilisation du modèle empirique pour estimer la valeur du runup exige la disponibilité des paramètres hydrodynamiques ( $\mathrm{H} 1 / 3$ et Lo au large) et morphologiques. Selon Enjalbert et al. (2012), la dynamique du runup est tributaire des paramètres hydrodynamiques (conditions de vagues) et environnementaux (paramètres morphologiques). Ces derniers constituent un paramètre déterminant dans la quantification du runup et les conditions d'utilisation dépendent des caractéristiques de la côte. Selon Cariolet (2011), sur les côtes microtidales comme celle faisant l'objet de cette étude, la variable morphologique utilisée pour estimer le runup doit se situer de la section de la plage comprise entre la limite de la pleine mer de vive eau (PMVE) et celle de la basse mer de vive eau (BMVE) (figure 4).

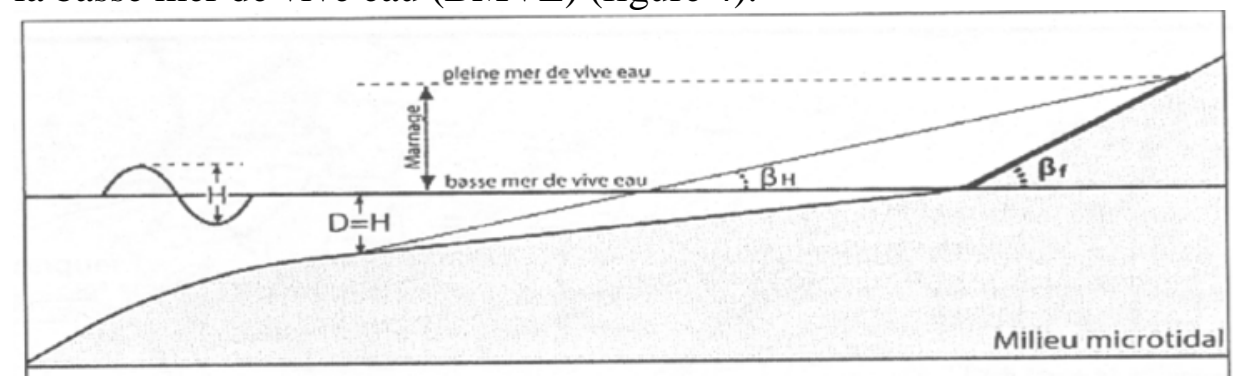

Figure 4: Représentation schématique de la pente dans le calcul du run up du microtidal (Cariolet, 2011)

En fonction des valeurs de pentes et de nombre d'Irribaren obtenus, une seule formule est utilisée. D’après Cariolet (2011), le choix d'un modèle empirique doit se fonder sur les caractéristiques des paramètres morphologiques de la plage (nombre d'Irribaren ${ }^{\varepsilon 0}$ ). Le nombre d'Irribaren des plages a été calculé à partir de la formule de Battjes (1974):

$$
\xi \circ=\frac{\tan \beta}{\left(\mathrm{Ho} / \mathrm{Lo}^{1} / 2\right.} \xi_{0}=\frac{\tan \beta}{\left(\mathrm{Ho} / \mathrm{Lo}^{1 / 2} / 2\right.}
$$

Lorsque $\boldsymbol{\xi} \mathbf{0}<\mathbf{0 , 5}$ la plage est dissipative car la pente est faible. Ce type de plage est caractérisé par un déferlement glissant. Autrement dit, la crête de la vague provoque sur sa face avant un mélange d'eau est d'air avant son déferlement (Bonte, 2013).

Si par contre, le nombre d'Irribaren est compris entre $\mathbf{0 , 5}<\boldsymbol{\xi} \mathbf{0}<\mathbf{2 , 5}$, le déferlement est plongeant. La crête de la vague est ainsi projetée vers l'avant et impacte violement la surface de l'eau.

Si le $\boldsymbol{\xi} \mathbf{0}>\mathbf{2 , 5}$, la pente de la plage est forte et dite réflective. Le déferlement est par conséquent gonflant ou frontal. La particularité est que sur les plages dites réflectives, la vague ne se déferle pas mais elle s’étale plutôt sur la plage. 
Tableau 1: Paramètres morphologiques des plages de Malibu, Gadaye et Malika en Octobre 2018

\begin{tabular}{|c|c|c|c|}
\hline Sites & Pente de l'estran $\left(^{\circ}\right)$ & Tan $\boldsymbol{\beta}$ & Nbre d'Irribaren \\
\hline Malibou & 4,22 & 0,073786367 & 0,82845725 \\
\hline Gadaye & 4,23 & 0,073961852 & 0,83042756 \\
\hline Malika & 4,16 & 0,072733549 & 0,81663644 \\
\hline
\end{tabular}

Pour les paramètres hydrodynamiques comme indiqué précédemment, les données de réanalyse de houle au large (hauteur significative et période de pic) de l'ANACIM ont été utilisées (tableau 2).

Tableau 2: Paramètres hydrodynamiques au large pendant la tempête du 19 novembre 2018

\begin{tabular}{|c|c|c|}
\hline $\begin{array}{c}\text { Hauteur significative au } \\
\text { large (Ho) }\end{array}$ & $\begin{array}{c}\text { Période de } \\
\text { Pic (T) }\end{array}$ & $\begin{array}{c}\text { Longueur d'onde au } \\
\text { large (Lo) }\end{array}$ \\
\hline 3,17 & 16 & 399,62 \\
\hline
\end{tabular}

Selon Laffitte (2018), au large la longueur d'onde des houles longues peut s'estimer par $\lambda=1,561 \mathrm{~T}^{2}=\mathrm{CT}$, donc $\mathrm{CT}=1,561 \mathrm{~T}$. Cependant, la longueur d'onde au large à partir de la formule suivante a été calculée :

$$
L o=1,561 m . s^{2} * T^{2}
$$

Pour les plages dont la pente $(\tan \beta)$ est comprise entre 0,026 et 0,14 , la formule de Holman (1986) et Nielsen et Hanslow (1991) a été appliquée :

$$
\begin{aligned}
& R 2 \%=0,92 \mathrm{Ho}_{\mathrm{O}} \\
& R \max =1,07 \mathrm{Ho} \xi_{\mathrm{o}}
\end{aligned}
$$

R2\% correspond à la valeur dépassée par $2 \%$ des plus hauts jets de rive. Rmax correspond à la valeur maximale atteint par le run up. 0,92 et 1,07 sont des coefficients sans dimension proposés par Holman (1986) Hanslow et Nielsen (1991). Ho est la hauteur significative au large Eo est le nombre d'Irribaren.

$\xi_{0}=$ le nonbre d’Iribarren. Il est calculé à partir de l'équation (1)

\section{Résultats}

\subsection{Description de la tempête du 18-19 novembre 2018}

Survenu un mois après la saison pluvieuse de 2018, cet événement est strictement d'origine marine. Il est ressenti sur toute l'étendue du secteur d'étude. De Malibu à Gadaye un vent d'une vitesse de $22 \mathrm{Km} / \mathrm{h}$ provenant de direction nord $\left(360^{\circ}\right)$ associé à une légère baisse de la pression atmosphérique estimée à $1012 \mathrm{hPa}$ aboutit à la submersion de ce linéaire côtier. Sur le plan marégraphique, cet événement est caractérisé par une hauteur d'eau considérable. En effet, les informations marégraphiques relevées au marégraphe de Dakar révèlent une importante hauteur de marée de l'ordre de $1,67 \mathrm{~m}$ à $6 \mathrm{~h}$ contre $1,59 \mathrm{~m}$ pour la marée prédite par le Service Hydrographique Océanographique de la Marine (SHOM). Cette différence 
entre la valeur de la marée prédite par le SHOM et la marée relevée par le marégraphe de Dakar se traduit par une surcote de 0,08 m (figure 5). En revanche, la hauteur maximale de l'onde de tempête est de $0,10 \mathrm{~m}$. Ainsi, ces conditions météorologiques et marégraphiques défavorables auxquelles s'ajoute un hydrodynamisme fort traduisent des conditions hydrodynamiques relativement énergétiques. En effet, les données de réanalyse de la houle au point d'extraction choisi révèlent une hauteur maximale de 3,16 m de direction NW avec une période de $16 \mathrm{~s}$ à $8 \mathrm{~h}$. Ainsi, la hauteur significative était supérieure ou égale à $2 \mathrm{~m}$ du 19 au 21 novembre à $5 \mathrm{~h}$ du matin. Cet événement météo-marin a affecté le linéaire côtier échantillon : Malibu, Gadaye et Malika.

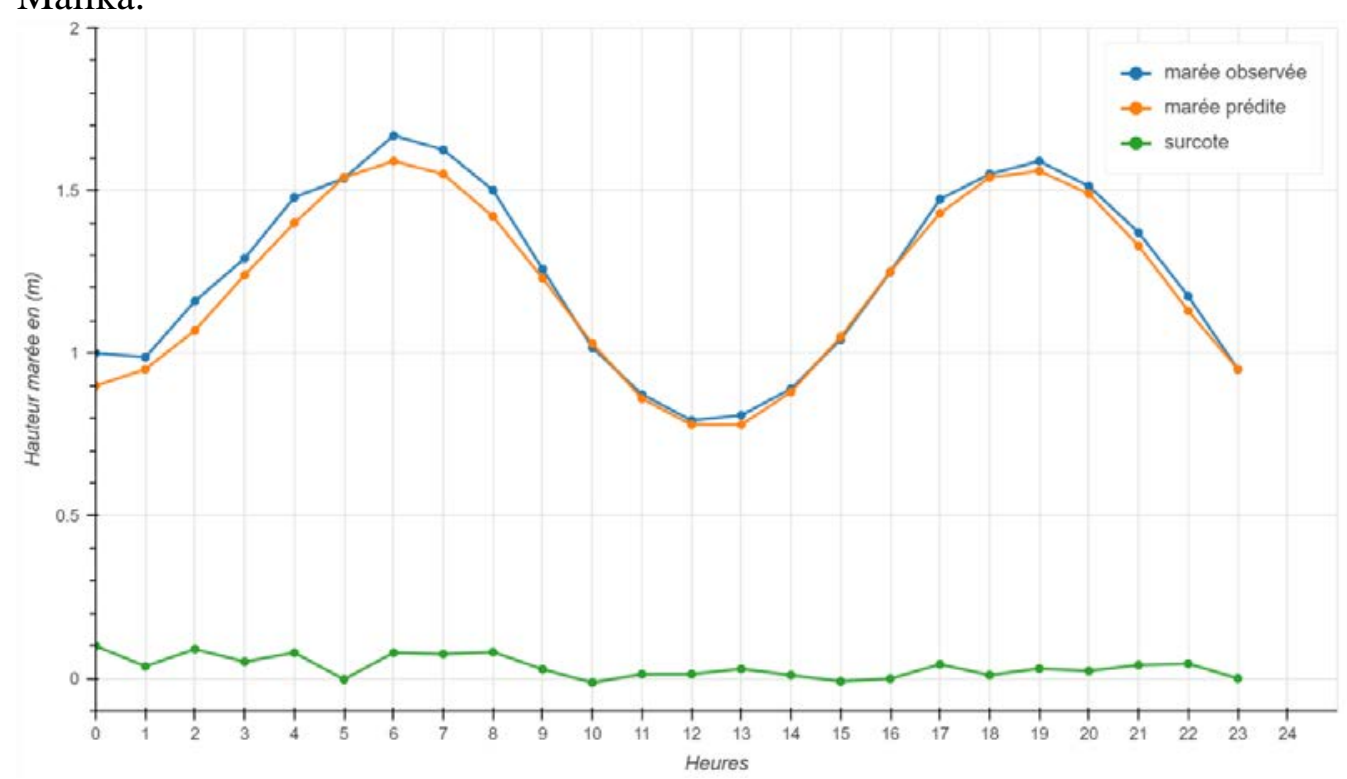

Figure 5 : Conditions marégraphiques de la submersion du 19 novembre 2018

\subsection{Niveaux d'eau extrêmes sur les plages de Malibu, Gadaye et Malika}

Le tableau 3 indique les niveaux d'eau extrêmes sur les plages de Malibu, Gadaye et Malika pendant la tempête de novembre 2018.

Tableau 3: Run up de la plage de Malibu, Gadaye et Malika

\begin{tabular}{|c|c|c|c|c|c|c|c|c|}
\hline \multirow{2}{*}{ Profil } & \multirow{2}{*}{$\begin{array}{c}\text { Niveau } \\
\text { marin }\end{array}$} & \multirow{2}{*}{ Ho } & \multirow{2}{*}{$\mathrm{T}$} & Lo & \multicolumn{2}{|c|}{ R2\% } & \multicolumn{2}{|c|}{ Rmax } \\
\cline { 6 - 9 } & (Dakar) & & & & R2\% & NM+R2\% & Rmax & NM+Rmax \\
\hline Malibu & 0,69 & 3,17 & 16 & 399,62 & 2,41 & 3,10 & 2,81 & 3,52 \\
\hline Gadaye & 0,69 & 3,17 & 16 & 399,62 & 2,42 & 3,11 & 2,82 & 3,53 \\
\hline Malika & 0,69 & 3,17 & 16 & 399,62 & 2,38 & 3,07 & 2,77 & 3,48 \\
\hline
\end{tabular}

${ }^{1}$ Niveau marin converti en données géodésiques grâce à la constante de Wöppelmann et al. (2008). 
La combinaison des hauteurs de la marée observée au marégraphe de Dakar et du runup maximal a permis d'estimer les niveaux extrêmes atteints par les vagues de tempête après leur déferlement sur les plages. La lecture du tableau 3 laisse apparaitre clairement que le niveau extrême atteint par le jet de rive est sensiblement le même sur les trois plages étudiées : Malibu, Gadaye et Malika. Ce tableau récapitule les hauteurs d'eau extrêmes obtenues à partir de l'application du modèle empirique de Holman (1986) Hanslow et Nielsen (1991). La figure 6 souligne les caractéristiques morphologiques des plages de Malibou, Gadaye et Malika au mois d'octobre 2018, un mois avant la submersion.
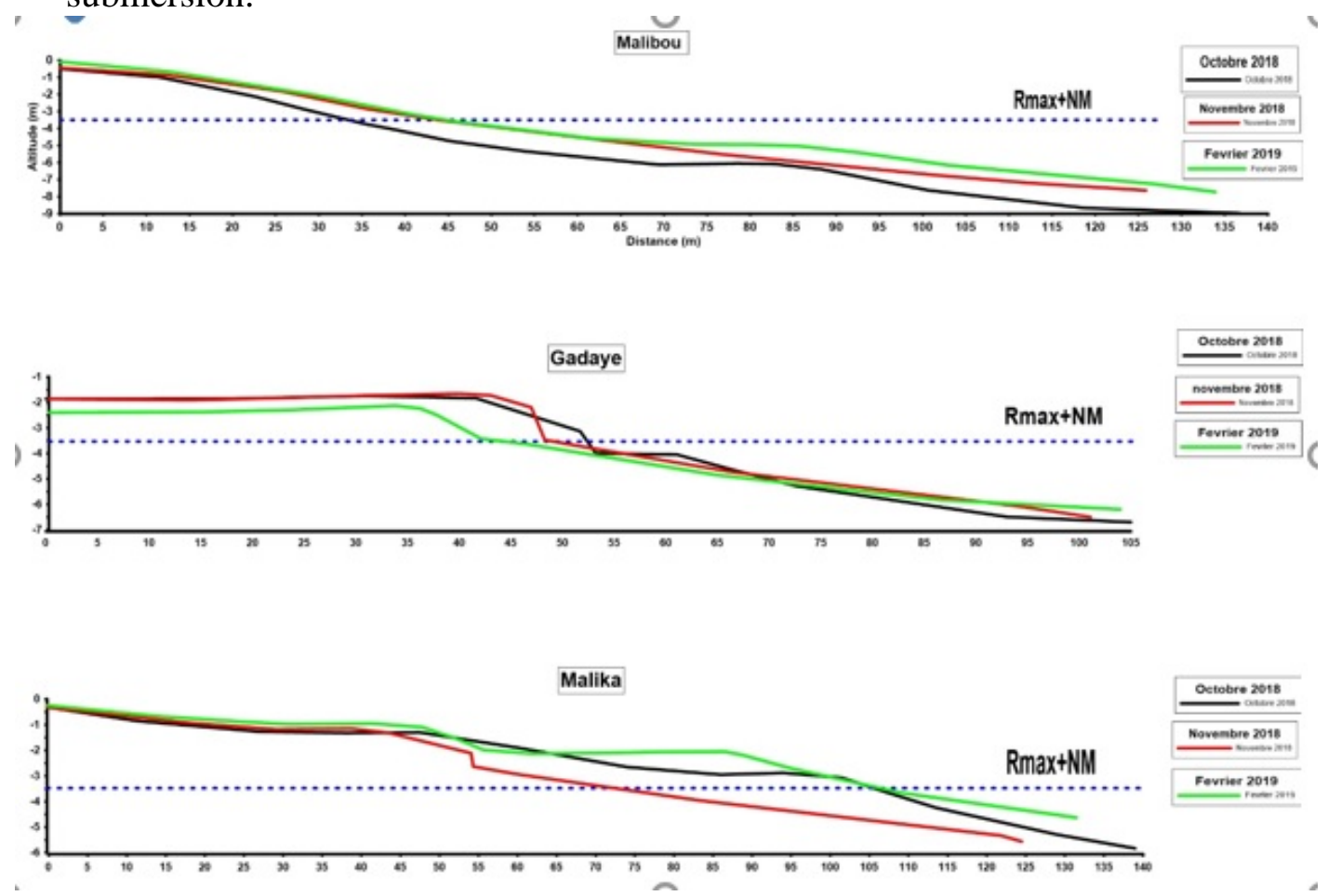

Figure 6: Caractéristiques morphologiques des plages de Malibu, Gadaye et Malika en octobre, novembre 2018 et février 2019

Cette même figure 5 met en exergue le potentiel de franchissement du trait de côte des plages de Malibu, Gadaye et Malika par les vagues de la tempête du 19 novembre 2018. En effet, la superposition du niveau d'eau maximale $(\mathrm{Rmax}+\mathrm{NM})$ avec les profils transversaux de la plage au mois d'octobre (un mois avant la tempête) révèle une absence de franchissement du trait de côte (figure 4), qui montre que les différents niveaux d'eau extrêmes enregistrés sur les trois plages (Malibu, Gadaye et Malika) n’ont pas dépassé la crête maximale du trait de côte respectif des trois plages. 


\subsection{Réponse morphosédimentaire des trois plages après la submersion du 19 novembre 2018}

Le tableau 4 indique les volumes sédimentaires au-dessus des niveaux d'eau extrêmes observés avant, pendant et après la submersion du 19 novembre 2018.

Tableau 4: Volumes sédimentaires au-dessus des niveaux d'eau extrêmes observés

\begin{tabular}{|c|c|c|c|}
\hline Date & $\begin{array}{c}\text { Malibu } \\
(\text { Rmax + NM = } \\
\text { 3,52m) }\end{array}$ & $\begin{array}{c}\text { Gadaye } \\
(\text { Rmax }+N M=3,5 \\
3 \mathbf{m})\end{array}$ & $\begin{array}{c}\text { Malika } \\
(\text { Rmax+NM }=3,4 \\
\text { 8m) }\end{array}$ \\
\hline \multicolumn{4}{|l|}{ Octobre 2018} \\
\hline $\begin{array}{c}\text { Volumes sédimentaires }\left(\mathrm{m}^{3}\right) \text { au- } \\
\text { dessus du Rmax+NM }\end{array}$ & 61,16 & 81.93 & 173,10 \\
\hline \multicolumn{4}{|l|}{ Novembre 2018} \\
\hline $\begin{array}{c}\text { Volumes sédimentaires }\left(\mathrm{m}^{3}\right) \text { au- } \\
\text { dessus du Rmax+NM }\end{array}$ & 61,27 & 77,03 & 157,26 \\
\hline \multicolumn{4}{|l|}{ Février 2019} \\
\hline $\begin{array}{c}\text { Volumes sédimentaires }\left(\mathrm{m}^{3}\right) \text { au- } \\
\text { dessus du Rmax+NM }\end{array}$ & 55,90 & 81,79 & 175,53 \\
\hline
\end{tabular}

La sensibilité des plages à la houle forte est évaluée par la comparaison des volumes de sédiments au-dessus des niveaux d'eau extrêmes entre octobre et novembre 2018. Celui de février 2019 a été calculé puis comparé à celui d'octobre 2018 (période de référence) pour évaluer le temps de retour des sédiments. Ce qui permet de se prononcer sur la résilience de ce segment côtier de Dakar aux tempêtes. L'analyse des résultats montre que la sensibilité des plages (Malibu, Gadaye et Malika) à l'action des vagues de tempête diffère d'une plage à une autre. Autrement dit, les plages présentent des caractéristiques morpho-sédimentaire diverses, voire hétérogènes. En effet, les fluctuations volumétriques au-dessus des niveaux d'eau extrêmes entre les différentes dates (octobre, novembre 2018 et février 2019) montrent une variabilité temporelle des caractéristiques morphologiques des plages selon les conditions hydrodynamiques.

\section{Discussion des résultats}

La superposition du niveau d'eau maximale (run up maximal et la marée observée) avec les profils transversaux de la plage au mois d'octobre et novembre 2018, puis février 2019 révèle une absence de franchissement du trait de côte. En effet, ces valeurs qui ne représentent que des ordres de grandeurs semblent bien refléter les observations de terrain après le passage de la tempête. En plus, les différents niveaux d'eau extrêmes enregistrés sur les trois plages (Malibu, Gadaye et Malika) n’ont pas dépassé la crête maximale du trait de côte respectif des trois plages. Autrement dit, le potentiel de franchissement des vagues au trait de côte est très faible, mais une submersion par rupture du cordon dunaire engendrée par l'écoulement 
tumultueux des eaux marines après le déferlement des vagues sur la plage peut occasionner une submersion de l'arrière-plage. Cela est conforme aux observations in situ (photo 1) le lendemain de la tempête (Sagne et al., 2020).

Photo 1 : Inondation de l'arrière plage pendant les houles fortes

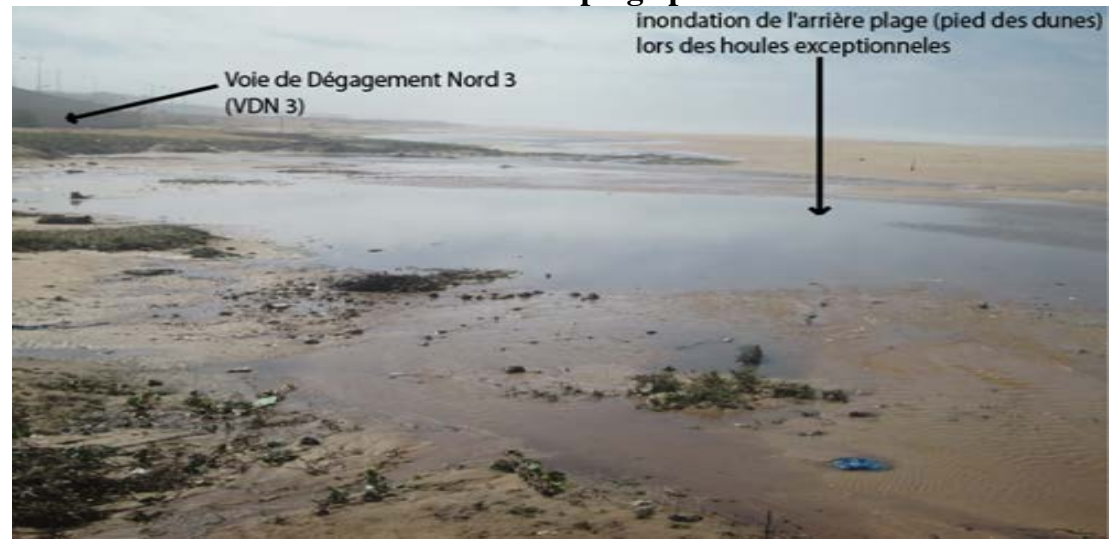

Source : Sagne et al., 2020)

Ce constant est fait aussi par Suanez et Fichaut (2010) sur les plages de l'ile de Banneg (archipel de Molène, Finistère, France). Sur la Langue de Barbarie, des destructions d'infrastructures par les houles fortes ont été notées en 2021 (photo 2), traduisant ainsi la recrudescence de ces phénomènes sur les côtes sénégalaises, d’où l'intérêt de promouvoir leur modélisation.

Photo 2. Destruction de l'École Cheikh Diop à Guet Ndar par la mer Images prises en octobre 2021 (SY B. A.)
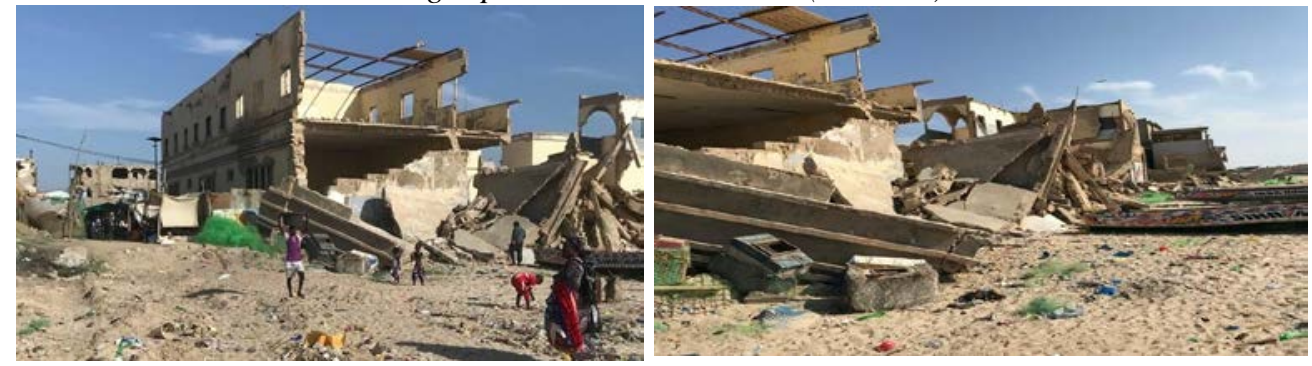

Même si les niveaux d'eau extrêmes sont sensiblement similaires sur les plages, le niveau de sensibilité ou d'exposition de cette zone à l'action du jet de rive diffère d'une plage à une autre. En effet, le niveau de la plage située en-dessous du niveau d'eau extrême est assez important sur la plage de Malibu. En revanche, sur les plages de Gadaye et Malika la distance séparant la limite maximale atteint par le jet de rive et le trait de côte est assez significative. La variation de la limite maximale du jet de rive sur les trois plages peut s'expliquer par leurs caractéristiques morphologiques distinctes. Une submersion n'est pas toujours homogène, la limite qu'elle peut atteindre dépend des variations morphologiques le long des côtes (Didier et al., 2020). A bien y regarder, la plage de Gadaye avant la tempête (octobre 2018) offrait 
une protection naturelle au trait de côte contre l'assaut des vagues due à la présence de morphotypes tels que la berme. La berme joue un rôle essentiel dans la protection du trait de côte. Ces structures morphologiques permettent de dissiper l'énergie des vagues avant que celles-ci n'atteignent le trait de côte (Bouvier, 2019). Selon Lerma et al. (2018), l'envergure des franchissements des vagues de tempête est fortement tributaire de la morphologie de la plage et du haut de plage. Car, la présence dans la zone intertidale d'un ilot rocheux ou d'une barre rocheuse protège les plages contre l'assaut des vagues de tempête (Quintin et al. 2013). Vu sa largeur et ses principales caractéristiques morphologiques marquées par la présence de berme, la plage de Gadaye semble être moins affectée par l'action des vagues de la tempête du 19 novembre 2018. Sur les plages larges possédant des bermes bien formées l'énergie de la houle de tempête est progressivement dissipée dans la zone de déferlement et sur l'estran (Balouin et al., 2011 ; Lerma et al., 2013). Naturellement comme tout évènement morphogénique, la tempête du 19 novembre 2018 a occasionné des impacts morphologiques sur les différentes plages à géométrie variable. Les vagues de tempête provoquent d'importantes modifications morphologiques (Stephan et al., 2010). Cette situation est la résultante de la morphologie des plages et de l'avant-côte avant l'évènement de submersion marine. La réponse morphologique de la plage à un évènement de forte énergie donné est tributaire de sa morphologie au moment de l'évènement (Castelle et al., 2010). Sur le plan morphologique et sédimentaire, la comparaison des profils levés au mois d'octobre et de novembre 2018 ont montré que les plages ont été inégalement impactées. Les variations morphologiques ont été observées quasiment sur toutes les plages mais, à des degrés divers. La morphologie d'une côte change si les sollicitations et les énergies appliquées varient (CETMEF, 2017). Les laisses de mer représentent la forme d'accumulation le plus visible occasionné par la tempête sur le plan morphologique. Cet évènement morphogène a en outre produit des formes d'ablation sur la partie haute des plages surtout celle de Gadaye et de Malika. Sur la partie supérieure de ces deux plages des microfalaises ont été modelées par l'action des vagues de tempête (photo 4). 
Photo 4 : Effets morphologiques des houles de tempêtes sur les plages de Gadaye et Malika (Sagne et al., 2020)
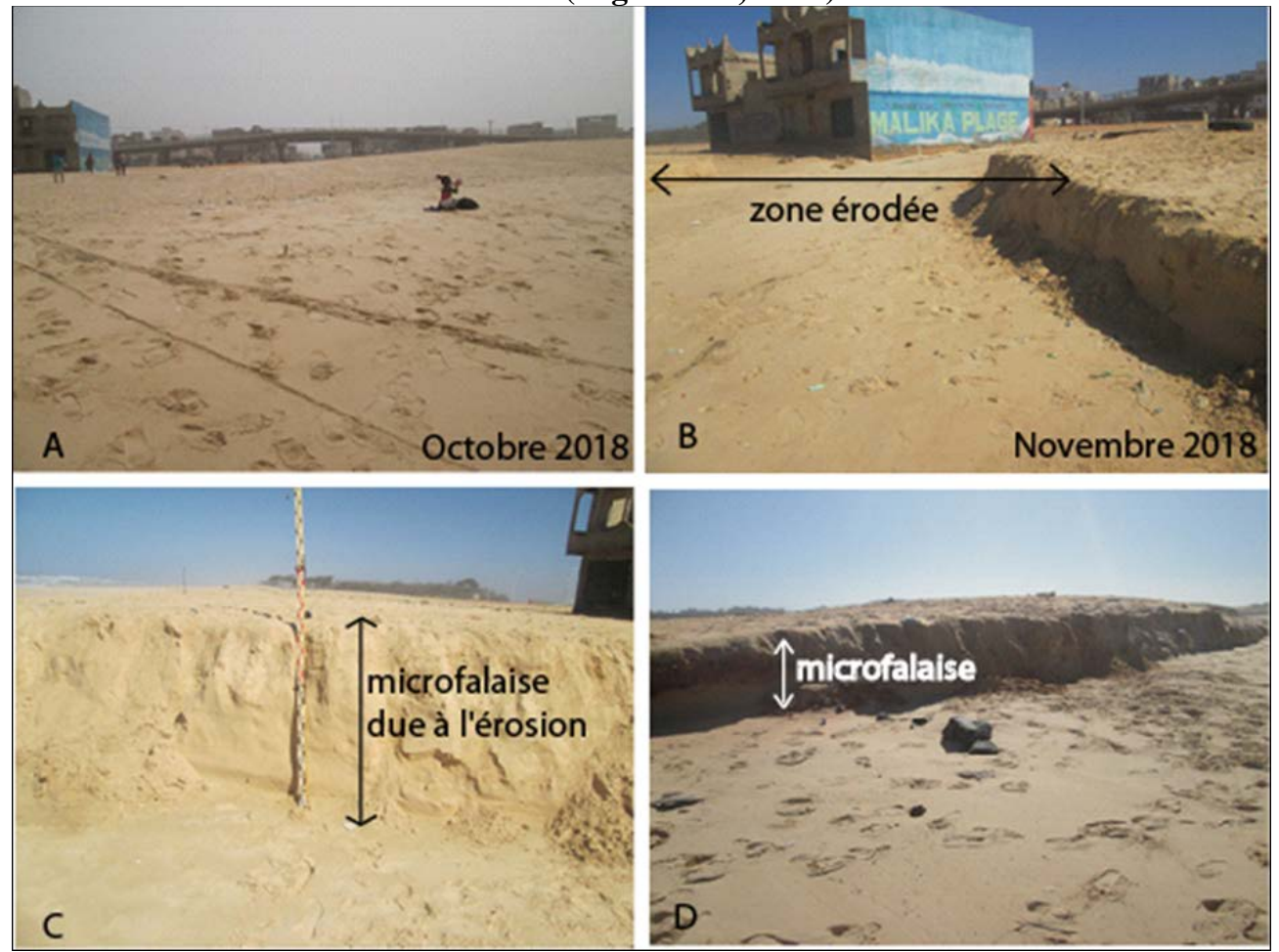

A: morphologie de la plage de Malika avant les houles de tempêtes, B: morphologie de la plage de Malika après les houles de tempêtes, C: microfalaise édifiée par les houles de tempêtes à la plage de Malika, D: microfalaise édifiée par les houles de tempêtes à la plage de Gadaye

Cette forme d'érosion s'explique par l'action morphogénique des niveaux d'eau extrêmes sur ces plages. Selon Bouvier (2019) les morphologies de la partie supérieure de la plage et du trait de côte sont conditionnées par l'action du jet de rive, lui-même est tributaire de la propagation des vagues dans le secteur d'avant-côte. Cette forme d'érosion (microfalaise) observée après le passage d'une tempête est aussi un indicateur de l'extension spatiale de l'onde de tempête sur la plage (Rey et al. 2017). Au-delà des impacts subis, les plages tendent à retrouver leur profil d'équilibre après le passage d'un épisode morphogénique. La lecture comparative des profils levés au mois d'octobre et novembre 2018, puis février 2019, révèle une certaine hétérogénéité dans les ajustements morphologiques et sédimentaires des plages de Malibu, Gadaye et Malika. En effet, trois mois après le passage de la tempête, nous avons observé que les plages de Malibu et de Malika ont connu un exhaussement rapide où les formes d'ablation observées au mois de novembre 2018 commençaient à disparaitre sous l'effet des conditions météo-marines clémentes et des apports sédimentaires. Cet engraissement des deux plages se 
traduit également par l'augmentation du volume sédimentaire au-dessus des niveaux d'eau extrêmes observés pendant la tempête. Cette situation traduit une certaine résilience de ce secteur du littoral nord de Dakar qui serait sans doute liée à la présence de stocks sédimentaires importants représentés par les dunes qui bordent la plage vers l'arrière. Ces dunes jouent un rôle crucial dans la capacité d'une côte à résister aux aléas côtiers que sont l'érosion et la submersion marine car alimentent les plages en sédiments et permettent de renouveler leur stock sédimentaire pendant les périodes de fortes houles érosives (Idier et Thiébot, 2013 ; Juinger et Robin, 2018). Cependant, elles commencent à subir une forte pression humaine qui se traduit par la coupe de filaos (Casuarina equisetifolia) pour faire place aux infrastructures routières (Voie de Dégagement Nord) et à usage d'habitations. Cette pratique pourrait entrainer la réduction du stock sédimentaire dunaire voire une disparition des dunes et la suppression de leurs rôles de protection naturelle contre l'érosion côtière surtout dans ce contexte actuel de changement climatique (Sagne et al., 2021). De ce fait, la protection et la régénération des filaos seraient très efficaces pour protéger cette zone des aléas climatiques.

\section{Conclusion}

Cette contribution a permis de faire la reconstitution des niveaux extrêmes sur les plages sableuses de Dakar (Malibu, Gadaye et Malika) après le passage de la tempête du 19 novembre 2018. En effet, les résultats montrent que les trois plages ont été inégalement impactées par la tempête et que la submersion n’a pas été subie de manière homogène sur les différents sites à l'étude. En sus, les effets géomorphologiques de cette tempête se sont traduits à travers les laisses de mer et les formes d'ablation telles que les microfalaises observées sur les plages après la tempête. Les résultats révèlent en outre que les plages n'ont pas le même niveau de résilience et d'ajustement morphosédimentaire face à un évènement morphogène à l'instar de la tempête du 19 novembre 2018. Cette étude a également apporté des informations nouvelles dans un domaine de recherche qui n'est pas suffisamment exploré en géomorphologie littorale sénégalaise. Au Sénégal, la plupart des thématiques de recherche concernant le littoral dudit pays porte sur le risque d'érosion côtière. En revanche, la question de la submersion marine et de la modélisation des niveaux extrêmes est très rarement abordée dans les travaux des géomorphologues et géologues littoralistes sénégalais. In fine, il est important de souligner que même si les résultats reflètent plus ou moins les observations faites sur le terrain, le modèle empirique utilisé dans le cadre de ce travail permet d'avoir des ordres de grandeurs sur l'extension maximale de l'onde de tempête sur les plages. La portion de la plage utilisée pour les paramètres morphologiques du modèle, le matériel utilisé pour les levers topomorphologiques (niveau de chantier) ainsi que les données d'altimétrie 
spatiale de la houle au large (données de réanalyse) peuvent aussi minimiser l'envergure de la submersion.

\section{References:}

1. Anselme B., Goeldner-Gianella L., et Durand P. (2008). Le risque de submersion dans le système lagunaire de La Palme (Languedoc, France) : nature de l'aléa et perception du risque, colloque international pluridisciplinaire Les littoraux : subir, dire et agir, Lille, France.

2. Arnaud G., et Bertin X. (2014). Contribution du setup induit par les vagues dans la surcote associée à la tempête Klaus, XIIIèmes Journées Nationales Génie Côtier Génie Civil Dunkerque, 2-4juillet, DOI :10.5150/jngcgc.2014.095-B (C) Editions Paralia CFL disponible en ligne - http://www.paralia.fr - available online.

3. Arnoux F., Abedie S., et Kojadinovic I. (2018). Analyse statistique du lien entre les variables d'aléa et les dommages par submersion, Vèmes Journées Nationales Génie Côtier - Génie Civil La Rochelle, 29 au $31 \mathrm{mai} 2018 \quad$ DOI :10.5150/jngcgc.2018.084 CEditionsParaliaCFLdisponibleenlignehttp://www.paralia.fr available online.

4. Balouin Y., Belon R., Delvallée E., Lamy M., et Bodere G., (2011). Etude complémentaire sur l'impact des tempêtes sur le littoral de la Plaine Orientale de Corse. Cartographie de la submersion marine de la sensibilité à l'érosion côtière et de l'exposition des enjeux. Rp-59724FR, 39 p+ volume cartographique.

5. Battjes J.A. (1974). Surf similarity, proceedings of the $14^{\text {th }}$ International Coastal Engineering Conference, American Society of Civil Engeneers, pp466-480.

6. Bouvier C., (2019). Barres d'avant côte et trait de côte : dynamique, couplage et effets induits par la mise en place d'un atténuateur de houle, thèse de doctorat physique de l'environnement, université de Bordeaux, $221 \mathrm{p}$.

7. Bulteau T., Delvallee E., Thiebot J., et Pedreros R. (2012). Retour d'expérience sur l'utilisation des probabilités conjointes pour la caractérisation de niveaux marins à la côte, XIIèmes Journées Nationales Génie Côtier Génie Civil Cherbourg,1214juinDOI:10.5150/jngcgc.2012.001-B (C) Editions Paralia CFL disponible en ligne - http://www.paralia.fr - available online.

8. Cariolet J.M.(2011). Quantification du runup sur une plage macrotidale à partir des conditions morphologiques et 
hydrodynamiques, Géomorphologie: relief, processus, environnement, $\mathrm{n}^{\circ} 1$, pp 95-108.

9. Caspar R., Costa S., et Jakob E.(2007). Fronts froids et submersions de tempête dans le nord-ouest de la France : le cas des inondations par la mer entre l'estuaire de la Seine et la baie de Somme, la Meteorologien57-mai, pp 37-47.

10. Castelle B., Marieu V., Bonneton P., et Bruneau N., (2010). Modélisation des profils de plage, la Houille Blanche $n^{\circ} 1$, pp $104-$ 110.

11. Didier D. (2020). Développement et analyse d'une méthode de cartographie de la submersion côtière dans l'estuaire et le Golfe du Saint-Laurent, QC, thèse de doctorat, Université du Québec à Rimouski, 318 p.

12. Enesser Y., Terrier M., et Said V.(2012). Changement climatique et risques naturels, l'Afrique, Terre de Connaissances, Géosciences_La revue du BRGM pour une terre durable $\mathrm{n}^{\circ} 21$, pp 50-57.

13. Enjalbert C., Senechal N., Bryan K.R., Coco G., Macmahan J., et Brown J. (2012). Dynamique de la zone de swash ; influence de la marée et de la morphologie sur les paramètres du run up, XIIèmes Journées Nationales Génie Côtier Génie Civil Cherbourg,1214juinDOI:10.5150/jngcgc.2012.006-E (C) Editions Paralia CFL disponible en ligne - http://www.paralia.fr - available online

14. Fall B.(2004 ). Le littoral de Yoff : évolution morphosédimentaire et changements à long terme de la ligne de rivage. Thèse $3^{\text {ème }}$ cycle, Université Cheikh Anta Diop de Dakar. 165 pp, 41 tab, 70 fig, 8 photos.

15. Faye I., 2010 : Dynamique du trait de côte sur les littoraux sableux de la Mauritanie à la Guinée-Bissau (Afrique de l'Ouest) : Approches régionale et locale par photo-interprétation, traitement d'images et analyse de cartes anciennes. Thèse de Doctorat, Université de Bretagne Occidentale, 393 p.

16. Garcin M., Desprats J.F., Fontaine M., Pedreros R., Sedan O., et Lenotre N. (2009). Risques côtiers à Sri Lanka : approches par GIS, scénarios et modélisation, la Houille blanche, $\mathrm{n}^{\circ} 1$, pp 54-58.

17. Gervais M., Balouin Y., Belon R., Certain R., Robin N., et Berne S., (2010). Impacts des tempêtes sur la morphologie d'un littoral microtidal : le site du Lido de Sète à Marseillan, Golfe du lion, XIèmes Journées Nationales Génie Côtier - Génie Civil Les Sables d'Olonne, 22-25 juin 2010 DOI:10.5150/jngcgc.2010.032-G (C) Editions Paralia CFL disponible en ligne http://www.paralia.fr - available online 
18. GIEC/IPCC, (2007) - Groupe de travail I du GIEC. Quatrième Rapport d'évaluation, Bilan 2007 des changements climatiques les bases scientifiques physiques, Résumé à l'intention des décideurs 25p.

19. GIEC. (2013). Résumé à l'intention des décideurs, Changements climatiques 2013 : Les éléments scientifiques

20. Le Gozannet G, Amraoui N, et Baills Audrey (2015). Vulnérabilité et adaptation au changement climatique: L'apport des Géosciences, Géosciences, BRGM, pp.16-27.

21. Idier B., et Thiébot J.(2013). Dynamique des côtes sableuses et phénomènes d'auto-organisation, géosciences $n^{\circ} 17$ octobre, pp 37-43.

22. Holman R.A. (1986). Extreme value statistics for wave run-up on a natural beach, Coastal Engineering, vol. 9, 527- 544.

23. Jeuck S. (2018). Digues de protection contre les submersions : état de l'art et modélisation numérique aux volumes finis du franchissement fluvial et maritime, science de l'environnement, travail de fin d'étude pour le diplôme d'ingénieur, Université de Lyon, 91p.

24. Juinger M., et Robin M. (2018). Caractérisation de la morphologie des massifs dunaires de la région Pays de la Loire (France) face au risqué de submersion marine, Vertigo-la revue électronique en sciences de l'environnement, vol $18 \mathrm{n}^{\circ} 2$

25. Letortu P., Costa S., et Cantat O. (2012). Les submersions marines en manches orientale: approche inductive et naturaliste pour la caractérisation des facteurs responsables des inondations par la mer, climatologie vol.9, pp 31-56.

26. Lerma A.N., Thomas Y.F Durand P., Anselme B., et Carlos A.(2013). Modélisation de submersions marines et leur validation par observation vidéo à Carthagène des Indes, Colombie, Géomorphologie : Relief Processus Environnement, vol 19-n²4, pp 481-498.

27. Lerma A. N., Pedreros R., Bulteau T., et Balouin Y. (2018). Stratégies de modélisation des submersions par franchissement de paquets de mer, Journées Nationales Génie Côtier, Journées-Génie Civil (JNGCGC), La Rochelle, France, 9 p.

28. Ndour A. (2015). Evolution morpho-sédimentaire et impacts des ouvrages de protection sur le littoral de Rufisque, Petite côte, thèse de doctorat, Univ. Cheikh Anta Diop de Dakar, 243 p.

29. Nielsen P., Hanslow D.J. (1991). Wave runup distributions on natural beaches, Journal of Coastal Research, vol. 7, 1139-1152.

30. Niang-Diop I. (1995). L’érosion côtière sur la Petite-Côte du Sénégal à partir de l'exemple de Rufisque. Passé - Présent - Futur. Thèse Université, Angers, tome 1, 318 pp, 112 fig., 47 tab 
31. Ovono Z.M et Poitier P. (2019). Le risque inondation dans les petits versants côtiers urbain de Libreville (Gabon). Exemple d'Ogombié et d'Inongui, les cahiers nantais, pp 39-49.

32. Paskoff R., (1993). Côtes en danger, Masson, Paris, 250 pp, 50 fig.

33. Paskoff R., (1998). La crise des plages: pénurie de sédiments, Mappemonde 52 ; 11-15p., 2fig.

34. Pinot J. P., (1998). Gestion du littoral. Tome I- littoraux tempérés : côtes rocheuses et sableuses. Institut océanographique, Paris, 399 pp, 222 fig

35. Quintin C., Bernatchez P., Jolivet Y. (2013). Impacts de la tempête du 06 décembre 2010 sur les côtes du Bas-Saint-Laurent et de la baie des chaleurs. Laboratoire de dynamique et de gestion intégrée des zones côtières et Chaire de recherche en géoscience côtière, Université du Québec à Rimouski. Rapport remis au ministère de la Sécurité publique du Québec, Volume I : 48p. + Volume II : 170 p.

36. Rey T., Le Dé L., et Gilbert D. (2017). Leçons tirées du cyclone Pam au Vanuatu (Mélanésie) : aléas côtiers, crues éclairs et dommages, Géomorphologie : relief, processus, environnement, vol 23- ${ }^{\circ} 4$.

37. Robin P. (2013). Hydrodynamique extrême en mer près des côtes, thèse de doctorat en physique, université de Province Aix-Marseille I, $339 \mathrm{p}$.

38. Sagne P., Fall B., Ba K., Sow E.H., et Niang-Diop I. (2020): Impacts of the storm surges on sandy beaches: example of northern coast of Dakar (Senegal); EWASH \& TI Journal, Volume 4 Issue 1,

39. Sagne P., Ba K., Fall B., Youm J.P.M., Faye G., Sarr J.P.G et Sow E.H. (2021). Cartographie De La Dynamique Historique Du Trait De Côte Des Plages De Guédiawaye Et Malika (Dakar, Sénégal, EuropeanScientificJournal, ESJ,17(25),214. https://doi.org/10.19044/esj.2021.v17n25p214

40. Sagne P. (2021) : étude morphosédimentaire et historique du littoral de la région de Dakar (Sénégal) : cas des plages des Mamelles, Ouakam, Malibu, Gadaye et Malika. Thèse de Doctorat Unique, Université Cheikh Anta Diop de Dakar, 209 pp., 34 tab., 114fig.,

41. Stephan P., Suanez S., et Fichaut Bernard. (2010). Franchissement et migration des cordons de gallet par rollover. Impact de la tempête du 10 mars 2008 dans l'évolution récente du Sillon de Talbert (Cotesd'Armor, Bretagne, Norois n²15 vol 2, pp 59-75.

42. Suanez S., et Fichaut B. (2010). Suivi morpho-sédimentaire de la plage de la Greve Rose entre 2009 et 2010, rapport de recherche LETGBrest UMR 6554 CNRS, Université de Bretagne Occidentale, 11p.

43. Stéphanain A., Balouin Y., Belon R., et Bodéré G. (2011) : Etude complémentaire sur le littoral de la plaine Orientale de Corse - état des 
connaissances sur les impacts des tempêtes sur le littoral. Rapport final du BRGM RP- 59058-FR 137pp., 64 ill., 4 ann.

44. Tchindjang M., Mbevo F.P., et Bopda A. (2019). Une Afrique atlantique avec des villes sous l'eau! Construire des villes côtières sans inondations, construire la ville portuaire de demain en Afrique Atlantique, édition ecm, $30 \mathrm{p}$.

45. Thior M, Sy A. A, Cissé I, Sané T, Diéye El , B, Ba B, D, Solly B, Descroix L, (2021). " Approche cartographique de l'évolution du trait de côte dans l'estuaire de la Casamance » http : //mappemonde.mgm.fr/ 131, 2021, 17p

46. Thior M, Sané,T, Dièye, El B. Sy,O Cissokho, D. Ba, B .D, Descroix, $\mathrm{L}$.(2019). "Coastline dynamics of the northern Lower Casamance (Senegal) and southern Gambia littoral from 1968 to 2017, Journal of African Earth Sciences”, Volume160, 2019, 103611, ISSN1464343X, https:

//doi.org/10.1016/j.jafrearsci.2019.103611. (http://www.sciencedirect.com/science/article/pii/S1464343X193026 63.

47. Weissenberger S., Noblet M., Plante S., Chouinard O., Guillemot J., Aubé M., Meur-Ferec C., Michel-Guilloui E., Gaye N., Kane A., Kane C., Niang A., et Seck A. (2016). Changements climatiques, changements du littoral et évolution de la vulnérabilité côtière au fil du temps : comparaison de territoires français, Canadien et sénégalais, Vertigo - la revue électronique en sciences de l'environnement, Volume 16 numéro 3, Vulnérabilités environnementales : perspectives historiques, pp 2-33. URL http:// vertigo.revues.org/18050; DOI: 10.4000/vertigo.18050

48. Wöppelmann G., Miguez B.L, et Creach R. (2008). Tide gauge records at Dakar, Senegal (Africa) : towards a 100-years consistent sea-level time series, European Geophysical Union, General Assambly, Vienna, Austria, 1 p. 